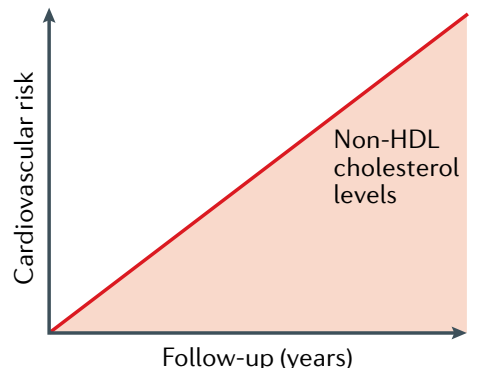

Credit: V.Summersby/Springer Nature Limited

probability of CVD events linked with non-HDL-cholesterol levels. The model predicted that a woman aged $<45$ years with a non-HDL-cholesterol level between 3.7 and $4.8 \mathrm{mmol} / \mathrm{l}$ plus two additional CVD risk factors had a $15.6 \%$ chance of experiencing a CVD event by the age of 75 years.

"Our data extend current know-

A stepwise increase in reported CVD events was observed across increasing concentrations of non-HDL cholesterol ledge and highlight the impact of nonHDL cholesterol on very long-term [CVD] outcomes," conclude the investigators. "These data could be useful for physician-patient communication about primary prevention strategies."

Karina Huynh

ORIGINAL ARTICLE Brunner, F. J. et al. Application of non-HDL cholesterol for population-based cardiovascular risk stratification: results from the Multinational Cardiovascular Risk Consortium. Lancet 394, 2173-2183 (2019)

GPR146 depletion are independent of LDLR activity.

"Development of LDL-C-lowering drugs acting independently of LDLR activity is urgently needed," remark the researchers. This unmet medical need is particularly relevant for patients with homozygous familial hypercholesterolaemia owing to $L D L R$ mutations, who have a suboptimal response to current LDL-C-lowering therapies such as statins and PCSK9 inhibitors, given that the effects of these agents require the upregulation of LDLR activity.

Yu and Cowan explain that further genetic studies are needed to confirm that GPR146 deficiency protects against hypercholesterolaemia and atherosclerotic cardiovascular disease in humans and highlight that the identification of the natural ligands of GPR146 will help uncover new mechanisms that regulate systemic cholesterol metabolism. "If successful, these studies would shed light on the development of therapies targeting GPR146," they comment. Irene Fernández-Ruiz depletion are independent of LDLR activity

ORIGINAL ARTICLE Yu, H. et al. GPR146 deficiency protects against hypercholesterolemia and atherosclerosis. Cell 179, 1276-1288.e14 (2019)

\section{CORONARY ARTERY DISEASE}

\section{Platelet-derived growth factor $A B$ promotes wound repair after $\mathrm{MI}$}

Intravenous infusion of recombinant human platelet-derived growth factor $\mathrm{AB}$ (rhPDGF-AB) in a porcine model of myocardial infarction (MI) promotes cardiac wound healing, improves cardiac function and increases early survival. These findings, published in Science Translational Medicine, indicate a strong translational potential for rhPDGF-AB as an adjunct to current Ml therapy.

Systemic infusion of rhPDGF-AB into a murine model of MI has previously been shown to improve cardiac function, which was associated with increased activation of fibroblasts. To extend these findings, James Chong and colleagues sought to determine whether rhPDGF-AB administered during the acute phase of wound healing after MI would modulate scar matrix remodelling. "We were keen to study these findings in a more clinically relevant animal model and, therefore, went on to the pig studies," explains Chong.

A total of 36 pigs were included in the study and assigned to undergo a sham procedure $(n=5)$ or Ml induced by angioplasty balloon inflation $(n=22)$. The pigs undergoing MI were further assigned in a 1:1 ratio to receive systemic rhPDGF-AB infusion or sterile water (vehicle) for 7 days via an osmotic mini-pump. Baseline measurements of infarct size (assessed by late gadolinium enhancement) and left ventricular ejection fraction (LVEF) were similar between both treatment groups. However, by day 28 , rhPDGF-AB-treated animals had an absolute increase in LVEF from baseline of $11.5 \pm 3.3 \%$ compared with a decrease of $3.5 \pm 1.5 \%$ in the vehicle-treated pigs. Furthermore, several indices of myocardial contractility including left ventricular end-systolic volume and the maximal rate of pressure change during systole all demonstrated improved cardiac contractility with rhPDGF-AB treatment.

In addition to functional improvements, rhPDGF-AB infusion was also associated with increased arteriogenesis and arteriolar branching, suggestive of increased de novo formation of microvessels. Surprisingly, rhPDGF-AB treatment did not reduce overall infarct scar size, but the collagen fibres in the scars of rhPDGF$\mathrm{AB}$-infused pigs were more aligned than those in the vehicle controls, signifying increased scar anisotropy, a conformation that has been associated with improved tissue mechanics. Finally, rhPDGF-AB treatment reduced early death associated with ventricular arrhythmias, possibly through decreased heterogeneity of post-infarct scar composition.

"One of the more important aspects of our findings is that the scar collagen matrix can be significantly modulated after MI," highlights Chong. "This observation has important potential implications for the development of future cardiac therapies." Chong and colleagues plan to perform more safety-focused experiments before progressing their work towards early-phase clinical testing.

Karina Huynh 